\title{
Steering Field Quality in the Main Dipole Magnets of the Large Hadron Collider
}

\author{
E. Todesco, B. Bellesia, L. Bottura, A. Devred, V. Remondino, S. Pauletta, S. Sanfilippo, W. Scandale, C. Vollinger, \\ and E. Wildner
}

\begin{abstract}
More than $10 \%$ of the collared coils of the main LHC dipoles have been produced. In this paper we compare the measured field quality to beam dynamics targets using correlations to measurements at $1.9 \mathrm{~K}$. The present status of field quality is given and corrective actions carried out to center field quality on optimal values are presented. Differences among the three manufacturers are analyzed, and the main results that concern correlation between cold and warm measurements are outlined. Present trends in the production and open points are discussed
\end{abstract}

Index Terms-Dipole magnets, field quality, superconducting accelerator magnets.

\section{INTRODUCTION}

$\mathbf{T}$ HE rate of production of the main dipoles of the Large Hadron Collider (LHC) [1] is ramping up and is now at about 20 magnets per month (about $2 / 3$ of the maximum production rate). These magnets are based on $\mathrm{NbTi}$ superconducting cable operated at $1.9 \mathrm{~K}$, and their field quality is mainly determined by the position of the conductors. Magnetic measurements are carried out in two steps: at room temperature at the various manufacturers, allowing to performing a sophisticated quality control [2] and to steering field quality, and at $1.9 \mathrm{~K}$ at CERN. The dependence of the field quality at room temperature (r.t) on the geometry of the dipole components is presented in [3].

Nearly 200 collared coils have been produced and 50 cold masses have been tested in operational conditions. With this wide set of data, we can assess with statistical significance the main issues of measured field quality versus the tight tolerances imposed by beam dynamics [4], singling out the most salient parameters. The analysis must rely on all measurements (both at $300 \mathrm{~K}$ and at $1.9 \mathrm{~K}$ ) to be able to react as soon as possible if some parameters are shown to diverge from the targets. Methods and solutions adopted for the previous large productions of superconducting magnets [5]-[7] are used for the analysis. A sketch of the dipole cross-section is given in Fig. 1.

\section{Measurements, Design Changes}

The main LHC dipoles are measured at r.t. during the assembly phase in the collared coil (superconducting coils clamped in stainless steel collars) and in the cold mass (collared coil and iron yoke inside welded outer shell).

Manuscript received October 22, 2003.

The authors are with the CERN, Accelerator Technology Division, Geneva A. Devred is also with the CEA/DSM/DAPNIA/SACM, Gif-sur-Yvette Cedex, France (e-mail: ezio.todesco@cern.ch).

Digital Object Identifier 10.1109/TASC.2004.829039

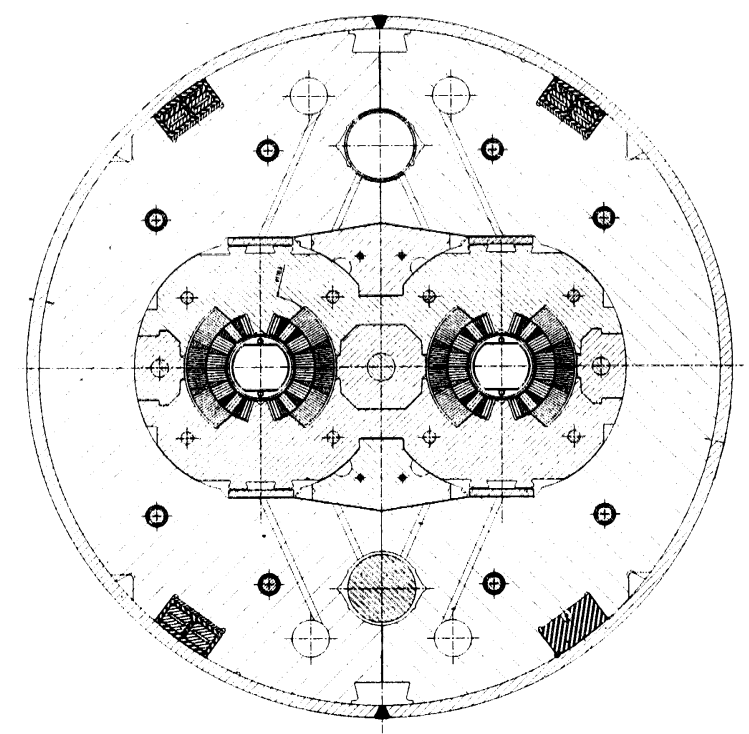

Fig. 1. Cross-section of the main LHC dipole.

The magnetic field is measured at r.t. with an excitation current ranging from \pm 8 to \pm 12 A with a $750 \mathrm{~mm}$ long rotating coil along 20 longitudinal positions to cover the total dipole length of $15 \mathrm{~m}$ [8]. Positions 1 and 20 are affected by the coil heads, and the positions 2 to 19 cover the so-called straight part. An average of measurements at positive and negative current is taken to cancel out the residual magnetization. Field quality is expressed according to the standard multipolar expansion

$$
B_{y}(x, y)+i B_{x}(x, y)=B_{0} \sum_{n=1}\left(b_{n}+i a_{n}\right) \frac{(x+i y)^{n-1}}{R_{r e f}^{n-1}} .
$$

where multipoles $\left(b_{\mathrm{n}}, a_{\mathrm{n}}\right)$ are given in $10^{-4}$ units. The reference radius $R_{\text {ref }}$ is fixed to $17 \mathrm{~mm}$. All the magnets will be measured at r.t. since this is a holding point of the production [2]. The magnets are later measured at CERN at $1.9 \mathrm{~K}$ with 12 rotating coils of $1.12 \mathrm{~m}$ length along the standard loadline from 760 to $11850 \mathrm{~A}$ and during a simulated machine cycle [8].

We have analyzed measurements of 184 collared coils and 122 cold masses at r.t, and 50 magnets at $1.9 \mathrm{~K}$. The share between manufacturer 1,2 , and 3 is $35 \%, 25 \%$ and $40 \%$ for measurements at r.t., and $50 \%, 25 \%$, and $25 \%$ at $1.9 \mathrm{~K}$. Design changes have been implemented to optimize the odd normal multipoles. The baseline of the coil lay-out (cross-section 1) as defined in [9] has been changed after 35 magnets ( 3 firms combined) through a modification of 0.1 to $0.4 \mathrm{~mm}$ in azimuthal size of the inner layer copper wedges, leaving unchanged the 
coil shape [10]. 135 magnets have been manufactured with the second lay out (denoted by cross-section 2 ), which has been then modified by increasing the mid-plane insulation (cross-section 3 , see Section VI-A).

In order to compensate variations of the azimuthal coil size, the thickness of shims between the coil pole and the collars are variable, giving a strong impact on multipoles [11]. In the first 30 magnets (3 firms combined), nonnominal shims have been used in $50 \%$ of the cases, with thickness differences up to $0.3 \mathrm{~mm}$. In the rest of the production, only $20 \%$ of collared coils feature nonnominal shims, with thickness differences of maximum $0.1 \mathrm{~mm}$. In this paper, data relative to odd multipoles are reduced to nominal shims to eliminate the bias due to this effect. This is done by subtracting from the measured values the expected effect of shim according to the sensitivity matrix presented in [11].

\section{Feedback Times And Methods for ANALysis}

Over the production of reference, the delay between a collared coil and a cold mass measurement (at r.t.) is in average 3 months, and between a cold mass measurement at r.t. and at $1.9 \mathrm{~K}$ is in average 7 months. The shortest time obtained from a collared coil measurement to a test at $1.9 \mathrm{~K}$ is 2.5 months. This shows that field quality analysis must rely on the collared coil data and on methods to extrapolate them in operational conditions.

Since any correction is acting only on the straight part of the collared coil, we start from collared coil averages over the straight part $b_{\mathrm{n}}^{\text {ccs. }}$. The effect of the iron yoke on multipoles is obtained by rescaling with a factor $k$ (i.e., the main field increase) plus an offset $b_{\mathrm{n}}^{\text {off, }} \mathrm{cm}$ [5]. We fix $k$ to the measured value of 1.18 (average over 122 cold masses), which is in good agreement with simulations. The offset $b_{\mathrm{n}}^{\text {off, }} \mathrm{cm}$ is computed from measurements

$$
b_{\mathrm{n}}^{\mathrm{off}, \mathrm{cm}}=b_{\mathrm{n}}^{\mathrm{cm}}-\frac{b_{\mathrm{n}}^{\mathrm{ccs}}}{k} .
$$

The same approach is used for computing the offsets from collared coil to high field $b_{\mathrm{n}}^{\text {off, high }}$ or to injection $b_{\mathrm{n}}^{\text {off,inj }}$

$$
b_{\mathrm{n}}^{\text {off }, \text { inj }}=b_{\mathrm{n}}^{\text {inj }}-\frac{b_{\mathrm{n}}^{\text {ccs }}}{k}, \quad b_{\mathrm{n}}^{\text {off,high }}=b_{\mathrm{n}}^{\text {high }}-\frac{b_{\mathrm{n}}^{\text {ccs }}}{k} .
$$

The injection values are the sum of the cold mass geometry and of the displacement on cool-down ("geometric"), plus the effect of persistent currents in superconductors. High field values are the sum of the geometric plus iron saturation and deformations due to Lorentz forces. In this approach, all effects are modeled as offsets to be applied to the cold mass data. Moreover, we assume that all offsets are independent of the manufacturer and therefore an average over all data is taken; this could give some bias since half of the magnets tested cold are from Firm1 (see Section II). This issue will be analyzed in Section VI-D.

For the magnetic length and for the main field, we have a correlation with slope one between collared coil and data at $1.9 \mathrm{~K}$, whose offset is calculated from the measurements.
TABLE I

SPREAD (1 SigMa, IN UNITS) IN FIELd QUALITY IN COLLARED CoILS RESCALED By $K$, CORRELATIONS TO OpERATIONAL CONDITIONS AND RANGES FOR SYSTEMATICS

\begin{tabular}{lccccccccccc}
\hline \hline & $\mathrm{L}$ & $\mathrm{B} / \mathrm{i}$ & $\mathrm{BL} / \mathrm{i}$ & $\mathrm{b} 2$ & $\mathrm{a} 2$ & $\mathrm{~b} 3$ & $\mathrm{a} 3$ & $\mathrm{~b} 4$ & $\mathrm{a} 4$ & $\mathrm{~b} 5$ & $\mathrm{~b} 7$ \\
\hline $\mathrm{cc} / \mathrm{k}$ & 3.0 & 7.3 & 8.3 & 0.55 & 1.08 & 0.96 & 0.39 & 0.09 & 0.29 & 0.38 & 0.12 \\
$\mathrm{high}-\mathrm{cc} / \mathrm{k}$ & 4.2 & 4.4 & 6.7 & 0.41 & 0.25 & 0.36 & 0.08 & 0.05 & $\mathbf{0 . 0 5}$ & 0.09 & 0.03 \\
inj/cc/k & 4.4 & 3.9 & 5.8 & 0.39 & $\mathbf{0 . 3 7}$ & 0.56 & 0.08 & 0.06 & $\mathbf{0 . 1 0}$ & $\mathbf{0 . 1 3}$ & 0.04 \\
\hline Target range & - & - & - & 3.60 & 1.70 & 6.00 & 3.00 & 0.85 & 0.22 & 0.65 & 0.40 \\
\hline \hline
\end{tabular}
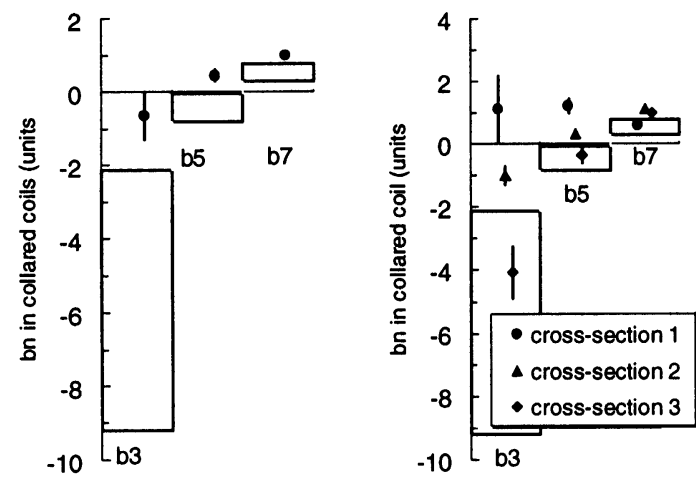

Fig. 2. Systematic multipoles measured in the straight part of 184 collared coil (markers) versus beam dynamics targets (solid lines) extrapolated using correlations to 50 measurements at $1.9 \mathrm{~K}$. Odd normal components: all data (left), and data separated according to different cross-sections (right).

\section{CORRElations AND STEERING OF Systematics}

The spreads of the components measured in the collared coils (rescaled by the factor $k$ ) are always larger than the spreads of the offsets from collared coil to high field $b_{\mathrm{n}}^{\text {off,high }}$ or to injec-

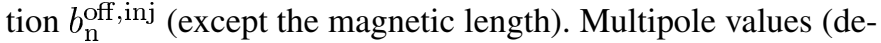
fined in Section II) are given in Table I. For magnetic length $\mathrm{L}$, transfer function $\mathrm{B} / \mathrm{i}$ and bending strength $\mathrm{BL} / \mathrm{i}$, we give the ratio between the spread and measured values, multiplied by $10^{4}$. The random part in operational conditions is determined by the collared coil. Correlations at injection field show that magnetization is controlled.

We also give a comparison with the allowed range for the systematic. These ranges are in general at least 10 times larger than the spread in the correlations and therefore collared coil values can be safely used to steer the systematic already with this set of correlations. The situation is more critical for $a_{2}, b_{5}$, and especially for $a_{4}$ (values in bold Table I), where the ratio between the systematic range and the spread is between 4 and 2 . All correlations should be anyway carefully monitored during the production.

\section{Measured Field Quality Versus Targets}

Since each manufacturer will provide $1 / 3$ of the magnets, the systematic component is defined as the average of the three averages relative to each manufacturer. Beam dynamic considerations provide target ranges for the systematics [4]. Skew and even normal components are within targets. Odd normal multipoles are given in Fig. 2, where in the left part the average of all data is considered, and in the right part we separated the three different coil cross-sections. Collared coils manufactured with the cross-section 3 feature $b_{3}$ and $b_{5}$ within targets, and $b_{7}$ at the 
TABLE II

EFFECT OF ADDITIONAL MidPLANE INSULATION (IN UNITS)

\begin{tabular}{|c|c|c|c|c|c|}
\hline & & $\mathrm{cl}$ & b3 & b5 & b7 \\
\hline \multirow{6}{*}{ Model } & A & -3.1 & -3.2 & -0.81 & -0.20 \\
\hline & $\mathrm{B}$ & -3.1 & -4.0 & -0.60 & -0.23 \\
\hline & Short & -7.9 & -3.5 & -0.52 & -0.18 \\
\hline & Firm1 (3 magnets) & -5.1 & -3.6 & -0.72 & -0.18 \\
\hline & Firm2 (4 magnets) & 0.1 & -2.5 & -0.66 & -0.15 \\
\hline & Firm3 (7 magnets) & -7.6 & -3.2 & -0.65 & -0.01 \\
\hline \multicolumn{2}{|c|}{ Measure average } & -4.2 & -3.1 & -0.68 & -0.12 \\
\hline
\end{tabular}

limit of the range (see also Section VI-A). The associated error bar is given with a 95\% confidence level (twice the standard deviation of the average).

The expected values of all randoms in operational conditions are within targets. The case of integrated main field is treated in Section VI-B.

\section{SPECIAL TOPICS}

\section{A. Corrective Action for Odd Normal Multipoles}

The implementation of cross-section 2 in March 2001 has considerably reduced the values of $b_{3}$ and $b_{5}$ [10]. These multipoles were marginally out of target ranges (see Fig. 2), and beam dynamics simulations showed this was not limiting the dynamic aperture. However, since the safety margin for $b_{3}, b_{5}$ and $b_{7}$ was narrow, a second change has been implemented in order to position these values at the center of the allowed band. At this stage of the production, the correction had to be minimal. Simulations have shown that the simple addition of $0.125 \mathrm{~mm}$ of insulation in the coil midplane would have brought both $b_{3}$ and $b_{5}$ on optimal values, and would have reduced $b_{7}$. This stripe of insulation is added on the midplane of either the upper or the lower pole, between the cable and the U-shaped insulation of $0.12 \mathrm{~mm}$ thickness. A similar solution was implemented in the RHIC magnets [7]. This correction does not imply a change of components and can be further adjusted during the production if required, thus giving an additional handle on field quality. A minor drawback is that the additional insulation increases coil prestress of $7 \mathrm{MPa}$, i.e., one quarter of the allowed range $\pm 15 \mathrm{MPa}$.

The change was tested on a $1 \mathrm{~m}$ long model, and then it was implemented on the series. Simulations and measurements are shown in Table II. We use a model A, i.e., where the additional midplane insulation is absorbed by a uniform azimuthal compression of the cables, collars and copper wedges are infinitely rigid, and all radial deformations are neglected. We then used a model B that takes into account of all deformations of collars and coil due to the forces evaluated through a finite element code. The same models were used for computing the influence of the pole shim size on the field quality [11]. A and B models agree within $25 \%$.

For the short magnet we consider the difference between two successive collarings that differ by $0.125 \mathrm{~mm}$ of insulation thickness. The error associated to this measurement is given by the reproducibility of field quality in different collarings, whose range according to measurements is 2 units of main field $c_{1}, 0.3$ of $b_{3}$ and 0.04 of $b_{5}$. Within these errors, the model B seems to give a better estimate of $b_{5}$, that is $25 \%$ lower than
TABLE III

Average DifFerence (In Units) Between Magnetic Length, Transfer FUNCTION (TF) AND INTEGRATED TF FOR EACH FIRM: THEIR RANGE $R$ and Standard Deviation, Correlations to High Field AND EXPECTED VALUES

\begin{tabular}{lccccccccc}
\hline \hline & \multicolumn{3}{c}{ Collared coil } & \multicolumn{3}{c}{ CC to high } & \multicolumn{3}{c}{ High expec } \\
& $\mathrm{L}$ & $\mathrm{B} / \mathrm{i}$ & $\mathrm{BL} / \mathrm{i}$ & \multicolumn{1}{c}{$\mathrm{L}$} & $\mathrm{B} / \mathrm{i}$ & $\mathrm{BL} / \mathrm{i}$ & \multicolumn{1}{c}{$\mathrm{L}$} & $\mathrm{B} / \mathrm{i}$ & $\mathrm{BL} / \mathrm{i}$ \\
\hline Firm1 ave & 2.3 & -1.6 & 0.7 & 0.1 & 0.9 & 1.0 & 2.4 & -0.7 & 1.7 \\
Firm2 ave & -0.4 & $\mathbf{- 5 . 6}$ & -6.0 & -1.2 & $\mathbf{2 . 4}$ & 1.2 & -1.6 & -3.2 & $\mathbf{- 4 . 8}$ \\
Firm3 ave & -1.9 & $\mathbf{7 . 1}$ & 5.2 & 1.0 & $\mathbf{- 3 . 2}$ & -2.2 & -0.9 & 3.9 & $\mathbf{3 . 0}$ \\
\hline R & 4.2 & 12.7 & 11.2 & 2.2 & 5.6 & 3.4 & 4.0 & 7.1 & 7.8 \\
sigma & 1.9 & 4.7 & & 3.8 & 4.3 & & & & \\
\hline \hline
\end{tabular}

what expected from model A. The same feature was observed for the pole shims [11]. For the series dipoles we obviously did not perform a recollaring of the same magnet and therefore we have to consider the difference between the average of cross-section 3 and cross-section 2 in each manufacturer. In this case the error is much larger due to the poor statistics (we have 14 cross-section 3 magnets): we can estimate the error as 6 units of $c_{1}, 1$ of $b_{3}$ and 0.4 of $b_{5}$ (95\% confidence level). Within these errors we have a good agreement, and the two models are not distinguishable. The low change of $b_{7}$ in Firm3 and of $c_{1}$ in Firm 2 could be due to a positive drift of both parameters that has been recently observed. More statistics is needed to assess these features.

\section{B. Spread in the Integrated Main Field}

Data relative to measurements of magnetic length $L$, transfer function $B / i$, and bending strength $(B L / i)$ are given in Table III. We compute the difference between the average of each firm with respect to the measured global mean value, and we divide it by measured values at high field. This relative spread is of the order of a few parts per 10000, and therefore is expressed in units of $10^{-4}$. For instance, from Table III, first row, we get 2 units for the magnetic length in Firm1: this means that $L$ in Firm 1 is $0.02 \%$ higher than the measured systematic.

In the collared coil we observe that Firm 3 has a transfer function of 7 units higher than average, whereas Firm2 is 6 units lower (Table III, bold values in collared coil columns).

This difference of 13 units ( 2.5 times the sigma in each firm) could correspond to a $0.13 \%$ difference in coil radial dimensions, which is close to construction tolerances (for instance, this gives 40 micron on inner radius).

Correlations from collared coil to high field are analyzed for all data (50 dipoles), assuming no influence of cross-section type. The increase in main field from collared coil to high field in Firm 3 is 6 units lower than in the Firms 1 and 2 (see Table III, bold values in "cc to high" columns). This partially compensates the previous effect. Since similar values are observed for correlations between collared coil and cold mass at r.t., this effect is due to the iron yoke. A difference of $0.15 \mathrm{~mm}$ in distance of the yoke laminations from the aperture center would give rise to this spread.

Summing the two contributions one can estimate that at high field, Firm 3 transfer function is 8 units higher than in Firms 1 and 2. Magnetic length shows a low spread between the firms, both in collared coil and in correlations (4 units), and therefore 
TABLE IV

AVERAGE Multipoles (IN UNITS), THEIR RANGE $(R)$, AND TyPICAL SPREAD AND RATIO OF RANGE TO SPREAD IN A COLLARED COIL

\begin{tabular}{lcccccccc}
\hline \hline CC & $\mathrm{b} 2$ & $\mathrm{a} 2$ & $\mathrm{~b} 3$ & $\mathrm{a} 3$ & $\mathrm{~b} 4$ & $\mathrm{a} 4$ & $\mathrm{~b} 5$ & $\mathrm{~b} 7$ \\
\hline Firm1 & -0.34 & 0.08 & -1.47 & -0.14 & -0.09 & -0.03 & $\mathbf{0 . 7 8}$ & $\mathbf{1 . 2 4}$ \\
Firm2 & -0.26 & 0.03 & -1.19 & $\mathbf{- 0 . 4 6}$ & -0.05 & 0.32 & 0.22 & $\mathbf{0 . 9 6}$ \\
Firm3 & -0.76 & 0.07 & -0.31 & $\mathbf{0 . 3 2}$ & -0.03 & -0.04 & $\mathbf{- 0 . 0 3}$ & 1.14 \\
\hline R & 0.51 & 0.05 & 1.16 & 0.78 & 0.06 & 0.36 & 0.80 & 0.27 \\
\hline sigma & 0.59 & 1.28 & 0.96 & 0.31 & 0.10 & 0.31 & 0.28 & 0.09 \\
R/sigma & 0.9 & 0.0 & 1.2 & 2.5 & 0.5 & 1.2 & 2.9 & 3.0 \\
\hline \hline
\end{tabular}

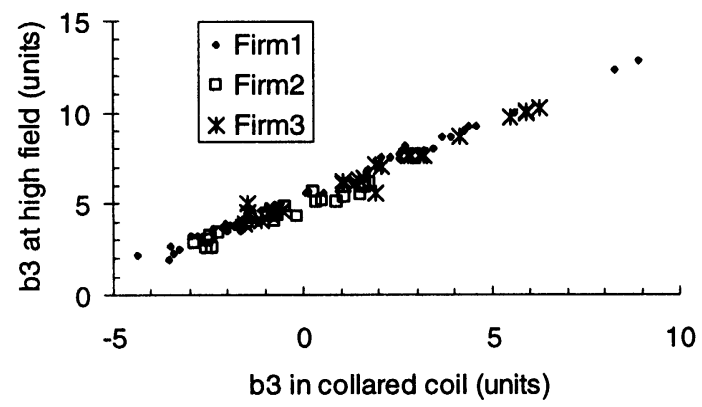

Fig. 3. $b_{3}$ in collared coil at r.t. versus values at high field at $1.9 \mathrm{~K}$.

the integrated transfer function average spread between firms is 7 units, i.e., within the 13 units target. If this spread would increase during the production, it could be corrected by varying the length of ferromagnetic laminations between the manufacturers. This solution has been already tested on a few magnets of Firm2.

\section{Differences Between Manufacturers: Collared Coil}

We first analyze the difference between firms in the average multipoles for the collared coils. We use all data for skew multipoles, data of Aperture 1 for $b_{2}$ and $b_{4}$ (the situation being similar for aperture 2), and data of cross-section 2 for $b_{3}, b_{5}$, and $b_{7}$. We compare the maximum difference $R$ between these averages to the typical spread of the manufacturer (average of the three sigma). Results are given in Table IV: we observe a negligible difference between firms in $a_{2}, b_{2}, b_{3}, b_{4}$, and $a_{4}$ ( $R$ equal to one sigma at most). Differences between firms are observed for $a_{3}$, $b_{5}$ and $b_{7}$ ( $R$ equal to around 3 sigma, bold values in Table IV). The most critical situation is for $b_{5}$ and $b_{7}$, since the range of the averages is as large as the systematic target range.

\section{Differences Between Manufacturers: Correlations}

We then analyze if there is a signature of the manufacturers

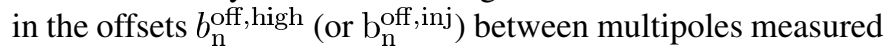
at high field (or injection) and the rescaled value of the collared coil $b_{\mathrm{n}}^{\text {ccs }} / k$ as defined in Section III. We observe either a negligible or a weak dependence of these offsets on the manufacturer. The case with higher dependence, where a ratio $R=1.8$ is observed, ( $b_{3}$ at high field) is shown in Fig. 3.

\section{CONCLUSION}

Systematics components are within targets, except $b_{7}$ that is on the upper limit. The increase of the midplane insulation to correct $b_{3} b_{5}$ and $b_{7}$ has been successful. Random components are within targets. The dependence of the field quality on the manufacturer is weak for the collared coils, and negligible for the correlations to operational conditions. This shows that the industrial processes are well optimized from a field quality point of view. The integrated main field spread is out of tolerance in the collared coils but is within targets at $1.9 \mathrm{~K}$. This parameter and odd normal multipoles should be carefully monitored during the production. We wish to thank all who contributed to perform measurements at the manufacturers and at CERN.

\section{REFERENCES}

[1] L. Rossi, , this conference.

[2] E. Wildner et al., "Production Follow-Up of the LHC Main Dipoles Through Magnetic Measurements at Room Temperature,", this proceedings.

[3] B. Bellesia et al., "Field Quality in the Main LHC Dipoles: Correlations Between Multipoles and Components in the Collared Coils,", this proceedings.

[4] S. Fartoukh and O. Bruning, "Field Quality Specification for the LHC Main Dipole Magnets,", LHC Project Report 501, 2001.

[5] P. Tollestrup, "The Amateur Magnets Builder Handbook,", FNAL report UPC86, 1979.

[6] A. Bonito Oliva, P. Gagliardi, R. Penco, and P. Valente, "A statistical analysis of the whole ansaldo HERA dipoles production," Cryogenics, vol. 30, pp. 589-593, September 1990.

[7] R. Gupta et al., "Field quality control through the production phase of RHIC arc dipoles," presented at the Particle Accelerator Conference, 1995.

[8] J. Billan et al., "Twin rotating coils for cold magnetic measurements of $15 \mathrm{~m}$ long LHC dipoles," IEEE Trans. Appl. Supercond., vol. 10, no. 1, pp. 1422-1426, March 2000.

[9] D. Perini et al., "Selection of the cross-section design for the LHC main dipole," IEEE Trans. Appl. Supercond., vol. 10, no. 1, pp. 65-68, March 2000.

[10] W. Scandale et al., "Controlling field quality in magnet production," presented at the Particle Accelerator Conference, 2003, also in CERN LHC-Project Report 659.

[11] P. Ferracin, W. Scandale, E. Todesco, and D. Tommasini, "Azimuthal coil size and field quality in the main CERN Large Hadron Collider dipoles," Phys. Rev. STAB, vol. 5, p. 62 401, 2001. 